\title{
Flexibility in a Stackelberg leadership with differentiated goods
}

\section{Fernanda A. Ferreira, Flávio Ferreira, Miguel Ferreira \& Alberto A. Pinto}

To cite this article: Fernanda A. Ferreira, Flávio Ferreira, Miguel Ferreira \& Alberto A. Pinto (2015) Flexibility in a Stackelberg leadership with differentiated goods, Optimization, 64:4, 877-893, DOI: 10.1080/02331934.2013.836649

To link to this article: https://doi.org/10.1080/02331934.2013.836649

$$
\text { 曲 Published online: } 20 \text { Sep } 2013 .
$$

Submit your article to this journal




\title{
Flexibility in a Stackelberg leadership with differentiated goods
}

\author{
Fernanda A. Ferreira $^{\mathrm{a}}$, Flávio Ferreira ${ }^{\mathrm{a}}$, Miguel Ferreira ${ }^{\mathrm{ab} *}$ and Alberto A. Pinto ${ }^{\mathrm{bc}}$ \\ ${ }^{a}$ ESEIG, Instituto Politécnico do Porto, Vila do Conde, Portugal; ${ }^{b}$ LIAAD - INESCTEC Porto L.A, \\ Porto, Portugal; ${ }^{c}$ Faculdade de Ciências da Universidade do Porto, Porto, Portugal
}

(Received 30 January 2013; accepted 20 July 2013)

\begin{abstract}
We study the effects of product differentiation in a Stackelberg model with demand uncertainty for the first mover. We do an ex-ante and ex-post analysis of the profits of the leader and of the follower firms in terms of product differentiation and of the demand uncertainty. We show that even with small uncertainty about the demand, the follower firm can achieve greater profits than the leader, if their products are sufficiently differentiated. We also compute the probability of the second firm having higher profit than the leading firm, subsequently showing the advantages and disadvantages of being either the leader or the follower firm.
\end{abstract}

Keywords: game theory; Stackelberg model; demand uncertainty; differentiation; perfect Bayesian equilibrium

AMS Subject Classifications: 91A15; 91A80

\section{Introduction}

The Stackelberg model [1] is one of the most widely used models in industrial organization to analyse the behaviour of the firms in a competitive environment. It models the strategic situation where firms sequentially choose their output levels in a market. The belief of firstmover advantage was widely held among entrepreneurs and venture capitalists, but is now questioned by numerous practitioners. There are examples of successful and unsuccessful pioneering firms as described, for instance, in Liu [2]: Dell was the first to introduce the direct-sale business model into the PC market, and it achieved great success; however, during the dot-com booming era, Pets.com, Webvan.com, Garden.com and eToys.com were all unsuccessful first movers in their respective market segments. The probability of success of pioneering in a market clearly depends on many factors, including technology, marketing strategy, market demand and product differentiation (see [1-18]). Liu [2] studied the effect of uncertainty in demand systems where only the leading firm is facing uncertainty in the demand parameter $\alpha$ that is considered to be uniformly distributed on an interval $\left[\alpha_{0}, \alpha_{1}\right]$. In this paper, we add the dimension of product differentiation to Liu's model and we show among other results that if their products are sufficiently differentiated, even with small uncertainty about the demand, the follower can achieve higher profits than the leader.

Our study focus on the influence of two parameters: the product differentiation $0<\gamma \leq 1$ and demand uncertainty $\theta \geq 1$. When $\gamma$ is close to one we have small

\footnotetext{
*Corresponding author. Email: miguelferreira@eu.ipp.pt
} 
differentiation between the goods and when $\gamma$ is small we have high differentiation between the goods. The demand uncertainty $\theta=\alpha_{1} / \alpha_{0}$ can be interpreted as the maximum value of demand using the minimum value of demand $\alpha_{0}$ as the unit. In Theorem 1 , we prove the existence of a unique perfect Bayesian equilibrium that requires a different reasoning from Liu's [2]. In Corollary 1, we compare the quantities produced by each firm and prove the existence of a threshold $T$ for $\alpha$, depending upon $\theta$ and $\gamma$, such that, bellow that threshold the leader is producing more than the follower and above that threshold the follower is producing more than the leader. In Theorem 2, we present the (ex-ante) expected profits of the two firms, depending upon the parameters $\theta$ and $\gamma$ and we characterize two parameter regions $A_{1}$ and $A_{2}$ in the parameter space $(\gamma, \theta)$ with the following properties: in the region $A_{1}$ the ex-ante profits of firm $F_{1}$ are higher than ex-ante profits of firm $F_{2}$; in the region $A_{2}$ the ex-ante profits of firm $F_{2}$ are higher than ex-ante profits of firm $F_{1}$. In Theorem 3 , we prove the existence of a threshold $\theta_{0}$ for the demand uncertainty parameter $\theta$, such that for $\theta$ greater than $\theta_{0}$ the expected profit of the follower is always (i.e. for any $\gamma$ ) greater than the expected profit of the leader. In Theorem 4, we find the (ex-post) profits of the firms and we characterize three parameter regions $P_{1}, P_{2}$ and $P_{3}$ in the parameter space $(\gamma, \theta)$ corresponding to three distinct economic behaviours: a region $P_{1}$, where the leading firm always has a higher profit than the follower firm; a region $P_{2}$, where there exists $R$, depending upon $\gamma$ and $\theta$, with the property that (i) if $\alpha<R$, the leading firm has a higher profit than the follower firm, and (ii) if $\alpha>R$, the follower firm has a higher profit than the leading firm; autoedited1. a region $P_{3}$, where there exists $L$, depending upon $\gamma$ and $\theta$, with the property that (i) if $L<\alpha<R$, the leading firm has a higher profit than the follower firm, and (ii) if $\alpha<L$ or $\alpha>R$, the follower firm has a higher profit than the leading firm. Hence, we show that the leading firm looses its advantage for high values of the demand intercept $\alpha$, if the demand uncertainty parameter $\theta$ belongs to the union of regions $P_{2}$ and $P_{3}$. Furthermore, the leading firm also looses its advantage for low values of the demand intercept $\alpha$, if the demand uncertainty parameter $\theta$ belongs to the region $P_{3}$. Furthermore, in Corollary 2, we compute the ex-ante probability $P\left(\pi_{2}^{*}>\pi_{1}^{*}\right)$ that the follower's ex-post profit is higher than the leader's ex-post profit. ex-ante expected profits and the ex-post profits of the leader firm and of the follower firm, and we also compute the probability $P\left(\pi_{2}^{*}>\pi_{1}^{*}\right)$ of the second firm having higher profit than the leading firm. We show that this probability increases both with the demand uncertainty and the degree of differentiation of the goods.

\section{The model and the perfect Bayesian equilibrium}

We start by describing the Stackelberg duopoly with product differentiation. We consider two firms, each producing a differentiated good. The demand, for simplicity, is linear

$$
\left\{\begin{array}{l}
p_{1}=\alpha-q_{1}-\gamma q_{2} \\
p_{2}=\alpha-\gamma q_{1}-q_{2}
\end{array},\right.
$$

with $\alpha>0$ and $0<\gamma \leq 1$, where $p_{i}$ is the price and $q_{i}$ the amount of good produced by the firm $F_{i}$, for $i \in\{1,2\}$. We note that the two products are substitutes and, since $\gamma \leq 1$, 'cross effects' are dominated by 'own effects'. The value of $\gamma$ expresses the degree of product differentiation. When $\gamma$ is equal to one, the goods are homogeneous, and when $\gamma$ tends to zero, we are close to independent goods (see [18]). We assume that the firms have the same constant marginal cost $c$. From now on, we consider prices net of marginal 
costs. This is without any loss of generality because if the marginal cost is positive, we may replace $\alpha$ by $\alpha-c$. We consider that the demand intercept is a random variable uniformly distributed in the interval $\left[\alpha_{0}, \alpha_{1}\right]$, with $\alpha_{1}>\alpha_{0}>0$. We note that, in this case, the demand uncertainty parameter $\theta$ is equal to the ratio $\alpha_{1} / \alpha_{0}$ (can be interpreted as the maximum value of demand using the minimum value of demand $\alpha_{0}$ as the unit). The distribution of $\alpha$ is common knowledge. Profit $\pi_{i}$ of firm $F_{i}$ is given by

$$
\pi_{i}=p_{i} q_{i}=\left(\alpha-q_{i}-\gamma q_{j}\right) q_{i} .
$$

As already stated in the Introduction, the timing of the game is as follows:

(i) Firm $F_{1}$ chooses a quantity level $q_{1} \geq 0$ without knowing the value of the demand realization;

(ii) Firm $F_{2}$ first observes the demand realization and observes $q_{1}$, and then chooses a quantity level $q_{2} \geq 0$.

In the next theorem, we show that this game has a unique perfect Bayesian equilibrium $\left(q_{1}^{*}, q_{2}^{*}\right)$ and we give its explicit characterization. Let

$$
K_{\gamma}=\frac{8-2 \gamma-3 \gamma^{2}}{\gamma(2-\gamma)},
$$

$A=\left(4-\gamma^{2}\right)^{2}-2 \gamma^{2}(4-3 \gamma), B=16\left(2-\gamma^{2}\right)$ and $C=2\left(8-3 \gamma^{3}\right)$, and denote by $\Delta$ the expression

$$
\Delta \equiv \Delta\left(\alpha_{0}, \gamma, \theta\right)=\alpha_{0}\left(4-2\left(2-\gamma^{2}\right) \theta+\sqrt{A \theta^{2}-B \theta+C}\right) .
$$

We observe that $A \alpha_{1}^{2}-B \alpha_{0} \alpha_{1}+C \alpha_{0}^{2} \geq 0$, for all $\theta=\alpha_{1} / \alpha_{0}>1$, and so $\Delta$ is well defined.

Theorem 1 Consider a differentiated products Stackelberg duopoly facing the demand system (1), where the parameter $\alpha$ is uniformly distributed in the interval $\left[\alpha_{0}, \alpha_{1}\right]$. Then, there is a unique perfect Bayesian equilibrium $\left(q_{1}^{*}, q_{2}^{*}\right)$ given as follows:

(i) If $\theta \leq K_{\gamma}$, then

$$
q_{1}^{*}=\frac{\alpha_{0}(2-\gamma)}{4\left(2-\gamma^{2}\right)}(\theta+1) \text { and } q_{2}^{*}=\frac{\alpha}{2}-\frac{\alpha_{0} \gamma(2-\gamma)}{8\left(2-\gamma^{2}\right)}(\theta+1) ;
$$

(ii) If $\theta \geq K_{\gamma}$, then

$$
q_{1}^{*}=\frac{\Delta}{3 \gamma^{3}} \text { and } q_{2}^{*}=\left\{\begin{array}{ll}
0, & \text { if } \alpha<\Delta /\left(3 \gamma^{2}\right) \\
\frac{\alpha}{2}-\frac{\Delta}{6 \gamma^{2}}, & \text { if } \alpha \geq \Delta /\left(3 \gamma^{2}\right)
\end{array} .\right.
$$

Theorem 1 is proved in the Appendix. From this theorem, we conclude that when the interval of $\alpha$ is large and the products are not very differentiated (large $\gamma$ ), if the realized demand is small the follower may choose not to produce at all. The relevant parameters in terms of their economical effects are $\gamma$ and $\theta$. In the next corollary, we compare the quantities produced by each firm. 
(a)

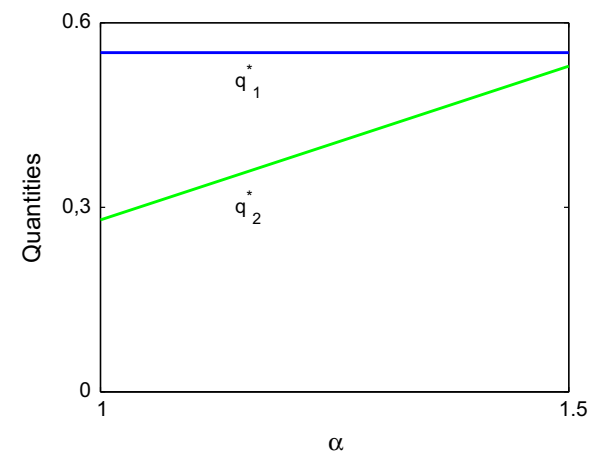

(b)

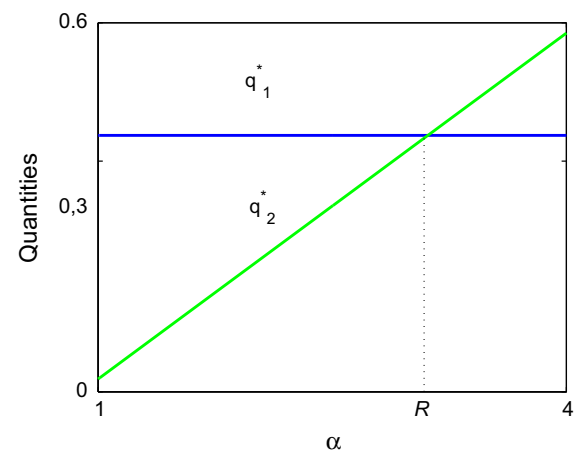

Figure 1. The quantities produced by each firm when the intervals of the uniform distribution of the parameter $\alpha$ are such that the ratio $\theta$ between its endpoints is as in each situation of Corollary 1, for $\gamma=0.8, \alpha_{0}=1$ and (a) $\alpha_{1}=1.5$; (b) $\alpha_{1}=4$.

Let $I_{\gamma}=(\gamma-4) /\left(3 \gamma^{2}-4\right)$.

Let

$$
R\left(\alpha_{0}, \gamma, \theta\right)= \begin{cases}R_{1}=\frac{\alpha_{0}\left(4-\gamma^{2}\right)}{4\left(2-\gamma^{2}\right)}(\theta+1) & \text { if } \theta \leq K_{\gamma} \\ R_{2}=\frac{(2+\gamma) \Delta}{3 \gamma^{3}} & \text { if } \theta \geq K_{\gamma}\end{cases}
$$

\section{Corollary 1}

(a) If $\theta<I_{\gamma}$, then, for all $\alpha \in\left[\alpha_{0}, \alpha_{1}\right]$, the leading firm produces more than firm $F_{2}$, i.e. $q_{2}^{*}<q_{1}^{*}$.

(b) If $\theta \geq I_{\gamma}$, then

(i) if $\alpha \leq R$, the leading firm produces more than firm $F_{2}$, i.e. $q_{2}^{*} \leq q_{1}^{*}$;

(ii) if $\alpha \geq R$, firm $F_{2}$ produces more than the leading firm, i.e. $q_{2}^{*} \geq q_{1}^{*}$.

Corollary 1 is proved in the Appendix. The three different situations described in this corollary are illustrated for some values of the parameters in Figure 1. We observe the existence of a threshold $R$ for $\alpha$ such that for values of $\alpha$ smaller than that threshold the leader is producing more, and for values of $\alpha$ greater than that threshold the follower is producing more. This is due to the fact that the leader makes the decision based only on expectations. The follower is going to produce more if the demand turns out to be large (and possibly more than the leader), and less if the realized demand is small (and possibly less than the leader).

\section{Ex-ante expected profits}

In this section, we present an ex-ante analysis of the Stackelberg game previously described. In the next theorem, we explicitly show the profits that the firms can expect, before the knowledge of the demand realization $\alpha$. For simplicity of notation, we denote by $\pi_{i}^{*}$ the profit $\pi_{i}\left(q_{1}^{*}, q_{2}^{*}\left(q_{1}^{*}, \alpha\right)\right)$, for $i \in\{1,2\}$. Let $\gamma_{0}:[3,+\infty) \rightarrow R_{0}^{+}$be defined by 


$$
\gamma_{0}(\theta)= \begin{cases}1, & \text { for } \theta=3 \\ \frac{1+\theta-\sqrt{\theta^{2}-6 \theta+25}}{\theta-3}, & \text { for } \theta>3\end{cases}
$$

Let

$$
Q=\left\{(\theta, \gamma): \theta \geq 3 \wedge \gamma_{0}(\theta) \leq \gamma \leq 1\right\}
$$

and

$$
P=([1,+\infty) \times(0,1]) \backslash Q .
$$

Let $\Sigma=\Delta / \alpha_{0}$.

THeOREM 2 While the demand realization $\alpha$ is unknown for both firms, their expected profits $E\left(\pi_{1}^{*}\right)$ and $E\left(\pi_{2}^{*}\right)$ are given by

$$
E\left(\pi_{1}^{*}\right)= \begin{cases}\frac{\alpha_{0}^{2}(2-\gamma)^{2}(\theta+1)^{2}}{32\left(2-\gamma^{2}\right)}, & \text { if }(\theta, \gamma) \in P \\ \frac{\alpha_{0} \Delta\left(18 \gamma^{3}\left(\theta^{2}-1\right)-12 \Sigma(\theta-1)-9 \gamma^{4} \theta^{2}+6 \gamma^{2} \Sigma \theta-\Sigma^{2}\right)}{108 \gamma^{6}(\theta-1)}, & \text { if }(\theta, \gamma) \in Q\end{cases}
$$

and

$$
E\left(\pi_{2}^{*}\right)= \begin{cases}\frac{\alpha_{0}^{2}\left(\left(7 \gamma^{4}+12 \gamma^{3}-28 \gamma^{2}-48 \gamma+64\right)\left(\theta^{2}+1\right)-2 \theta\left(\gamma^{4}-12 \gamma^{3}-4 \gamma^{2}+48 \gamma-32\right)\right)}{192\left(\gamma^{2}-2\right)^{2}}, & \text { if }(\theta, \gamma) \in P \\ \frac{\alpha_{0}^{2}\left(3 \gamma^{2} \theta-\Sigma\right)^{3}}{324 \gamma^{6}(\theta-1)}, & \text { if }(\theta, \gamma) \in Q\end{cases}
$$

Theorem 2 is proved in the Appendix.

Let $\alpha_{0}=1$. Let $A_{1}=\left\{(\gamma, \theta): E\left(\pi_{1}^{*}\right)>E\left(\pi_{2}^{*}\right)\right\}$ and $A_{2}=\left\{(\gamma, \theta): E\left(\pi_{2}^{*}\right)>E\left(\pi_{1}^{*}\right)\right\}$. Then, line $E\left(\pi_{1}^{*}(\alpha)\right)=E\left(\pi_{2}^{*}(\alpha)\right)$ is given by $C(\theta, \gamma)=0$, defined by

$$
C(\theta, \gamma)= \begin{cases}\theta-\frac{4 \gamma\left(2-\gamma^{2}\right) \sqrt{12 \gamma-9 \gamma^{2}}-5 \gamma^{4}+4\left(3 \gamma^{3}-4 \gamma^{2}+4\right)}{13 \gamma^{4}-12 \gamma^{3}-16 \gamma^{2}+16}, & \text { if }(\theta, \gamma) \in P \\ D \theta^{5}+E \theta^{4}+F \theta^{3}+2 G \theta^{2}+H \theta+49 \gamma^{3}-120, & \text { if }(\theta, \gamma) \in Q\end{cases}
$$

where $D=15 \gamma^{5}-36 \gamma^{4}+7 \gamma^{3}+42 \gamma^{2}-40, E=15 \gamma^{5}-6 \gamma^{4}+49 \gamma^{3}-138 \gamma^{2}+200$, $F=6 \gamma^{4}-56 \gamma^{3}+192 \gamma^{2}-400, G=3 \gamma^{4}-49 \gamma^{3}+120$ and $H=49 \gamma^{3}-96 \gamma^{2}+120$ (Figure 2).

THEOREM 3 There is $\theta_{0} \approx 7.30$ such that if the uncertainty parameter $\theta$ is greater than $\theta_{0}$, then the expected profit of the follower firm is always greater than the expected profit of the leading firm.

Theorem 3 is proved in the Appendix.

In Figure 3, we plot the firms' expected profits, $E\left(\pi_{1}^{*}\right)$ and $E\left(\pi_{2}^{*}\right)$, as functions of the demand uncertainty parameter $\theta$ and of the degree $\gamma$ of product differentiation. Figure 4 illustrates cross-sections of Figure 3 at the degrees $\gamma=0.9$ (Figure 4(a)) and $\gamma=0.5$ (Figure 4(b)) of product differentiation. Figure 5 illustrates cross-sections of Figure 3 at the demand uncertainty parameter's values $\theta=2$ (Figure 5(a)) and $\theta=8$ (Figure 5(b)). 


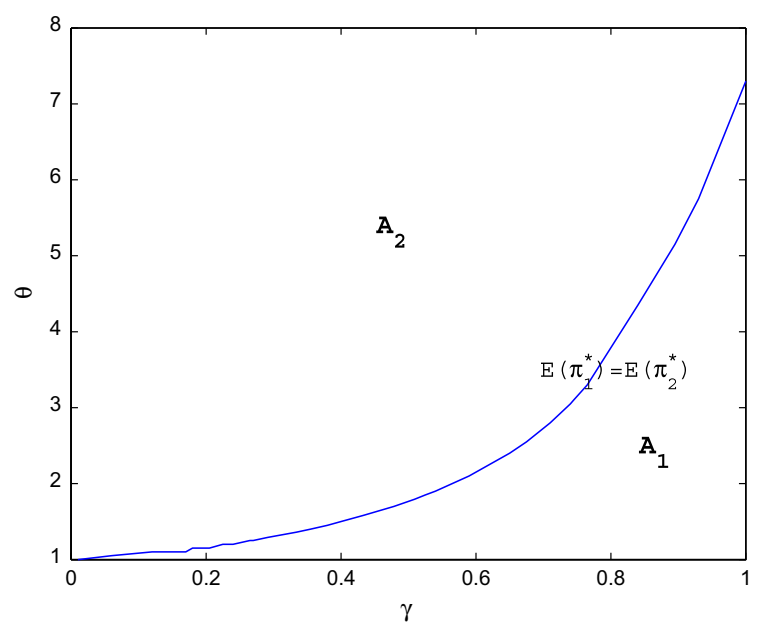

Figure 2. Plot of $C(\theta, \gamma)$.

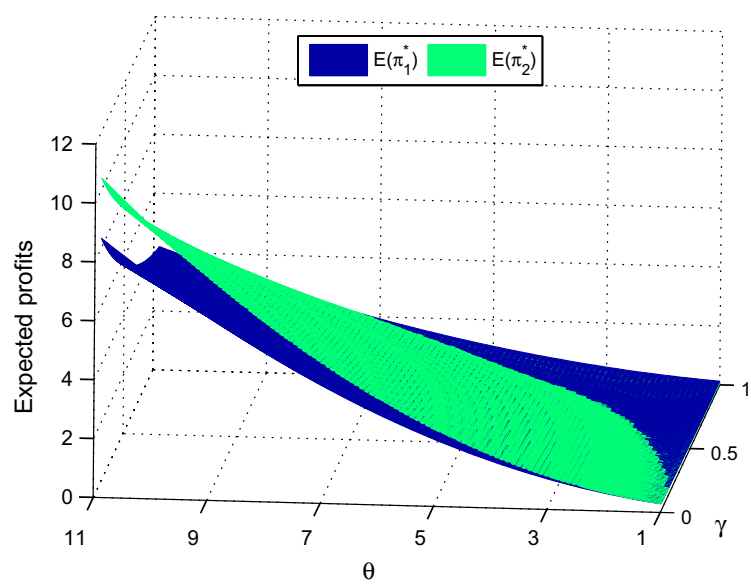

Figure 3. Firms' expected profits varying with the demand uncertainty parameter $\theta$ and with the degree $\gamma$ of the product differentiation, by taking $\alpha_{0}=1$.

These figures illustrate that both parameters $\theta$ and $\gamma$ are relevant to determine which firm has ex-ante higher expected profits.

\section{Ex-post profits}

In this section, we present the ex-post analysis of the same Stackelberg game. In Theorem 4, we explicitly present the ex-post profits $\pi_{1}^{*}$ and $\pi_{2}^{*}$ of firms 1 and 2, respectively, obtained after the observation of the demand realization. We describe three regions for the demand uncertainty parameter $\theta=\alpha_{1} / \alpha_{0}$ corresponding to three distinct profits relations between the leading and the follower firms (see Figure 6). The low-medium uncertainty boundary value is 
(a)

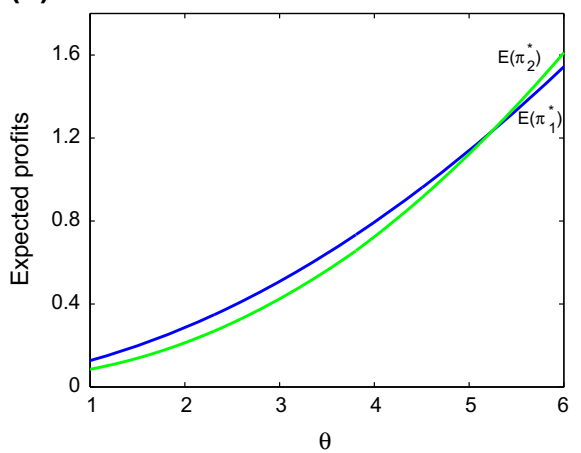

(b)

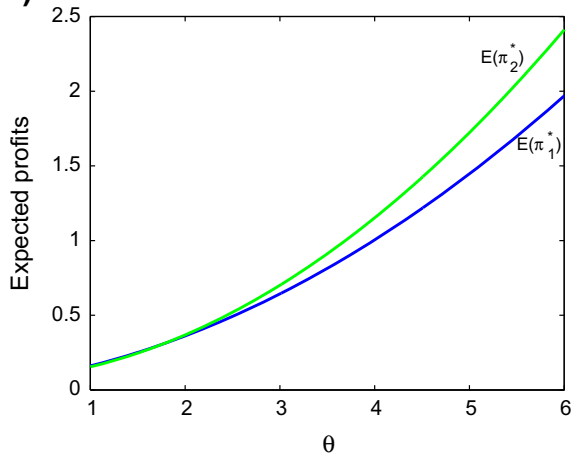

Figure 4. Cross-sections of Figure 3 at the degrees (a) $\gamma=0.9$ and (b) $\gamma=0.5$ of product differentiation.
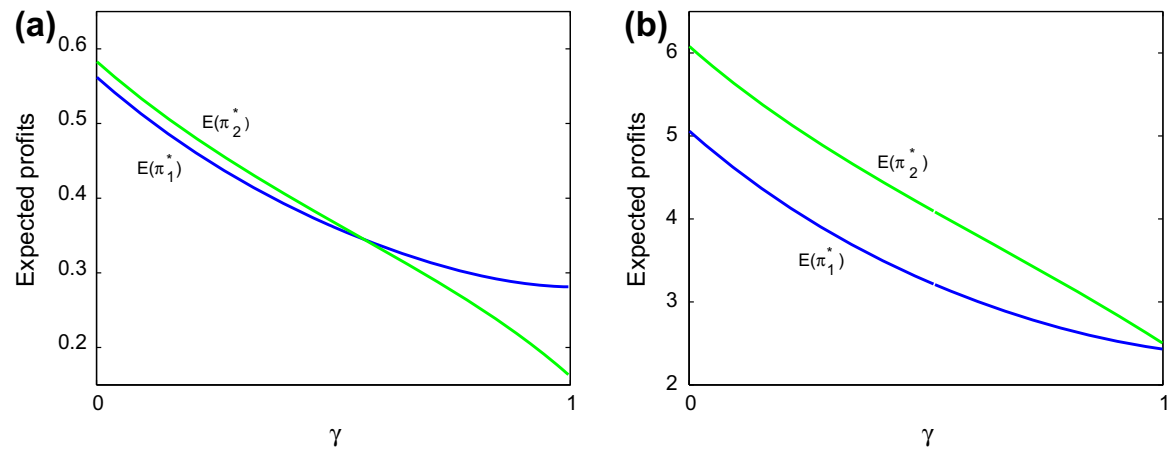

Figure 5. Cross-sections of Figure 3 at the uncertainty parameter's values (a) $\theta=2$ and (b) $\theta=8$.

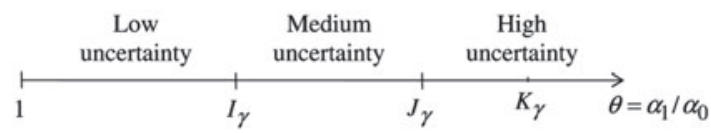

Figure 6. Three regions for the demand uncertainty parameter $\theta$ corresponding to three distinct profits relations between the leading and the follower firms.

$$
I_{\gamma}=\frac{4-\gamma^{2}}{4-3 \gamma^{2}},
$$

and the medium-high uncertainty boundary value is

$$
J_{\gamma}=\frac{4+4 \gamma-5 \gamma^{2}}{(2-\gamma)^{2}} .
$$




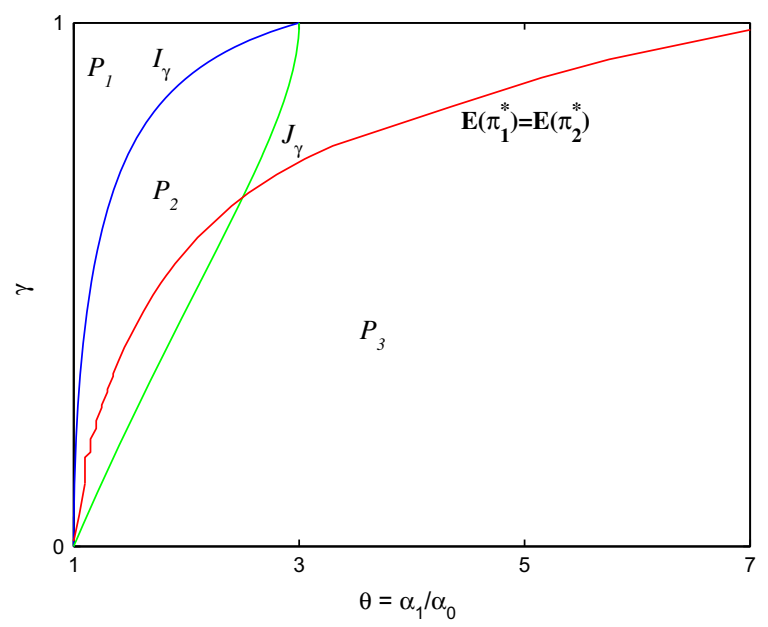

Figure 7. Plots of the functions $I_{\gamma}$ and $J_{\gamma}$, and the regions $P_{1}, P_{2}$ and $P_{3}$.

Let $P_{1}=\left\{(\theta, \gamma): \theta<I_{\gamma}\right\}, P_{2}=\left\{(\theta, \gamma): I_{\gamma} \leq \theta \leq J_{\gamma}\right\}$ and $P_{3}=\left\{(\theta, \gamma): \theta>J_{\gamma}\right\}$ (see Figure 7).

The functions $I_{\gamma}$ and $J_{\gamma}$ characterize the demand uncertainty parameter $\theta$ for which the leading firm looses its advantage for some realizations of the demand random variable. In fact, in the next theorem, we will show that the leading firm looses its advantage for high values of the demand intercept, if the demand uncertainty parameter $\theta$ is greater than $I_{\gamma}$, and also for low values of the demand intercept, if the demand uncertainty parameter $\theta$ is greater than $J_{\gamma}$. Hence, for high values of the demand uncertainty parameter $\left(\theta>J_{\gamma}\right)$ only in an intermediate zone of the realized demand does the first mover preserve its advantage. We observe that for homogeneous goods $(\gamma=1)$, the functions $I_{\gamma}$ and $J_{\gamma}$ coincide, i.e. $I_{1}=J_{1}$ (Liu's case [2]), and for non-homogeneous goods $(0 \leq \gamma<1)$, we have that $I_{\gamma}<J_{\gamma}$. Observe that the curve given by $E\left(\pi_{1}^{*}\right)=E\left(\pi_{2}^{*}\right)$ crosses the curves $P_{1}$ and $P_{2}$ (see Figure 7).

We define $L$ and $R$ as follows:

$$
L \equiv L\left(\alpha_{0}, \gamma, \theta\right)= \begin{cases}\frac{\alpha_{0}(2-\gamma)^{2}}{4\left(2-\gamma^{2}\right)}(\theta+1), & \text { if } \theta \leq K_{\gamma} \\ \frac{(2-\gamma) \Delta}{3 \gamma^{3}}, & \text { if } \theta \geq K_{\gamma}\end{cases}
$$

and

$$
R \equiv R\left(\alpha_{0}, \gamma, \theta\right)= \begin{cases}\frac{\alpha_{0}\left(4-\gamma^{2}\right)}{4\left(2-\gamma^{2}\right)}(\theta+1), & \text { if } \theta \leq K_{\gamma} \\ \frac{(2+\gamma) \Delta}{3 \gamma^{3}}, & \text { if } \theta \geq K_{\gamma}\end{cases}
$$

THEOREM 4

(a) Case $\theta \leq I_{\gamma}$. For all $\alpha \in\left[\alpha_{0}, \alpha_{1}\right]$, the leading firm has a higher profit than firm $F_{2}$, i.e. $\pi_{2}^{*}<\pi_{1}^{*}$.

(b) Case $I_{\gamma}<\theta \leq J_{\gamma}$. The value $R \in\left(\alpha_{0}, \alpha_{1}\right)$. Furthermore, 
(a)

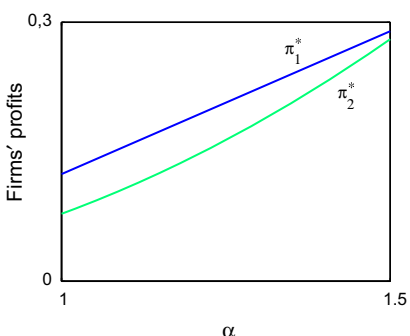

(b)

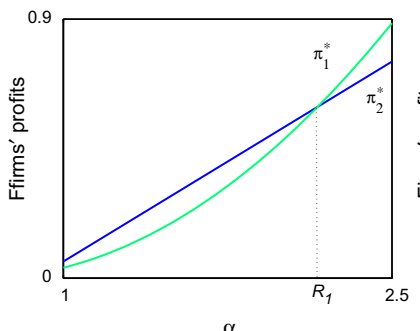

(c)

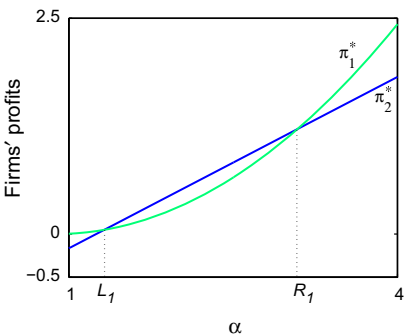

Figure 8. The profits of both firms when the intervals of the uniform distribution of the parameter $\alpha$ are such that the ratio $\theta$ between its endpoints is as in each situation of Theorem 4 , for $\gamma=0.8$, $\alpha_{0}=1$ and (a) $\alpha_{1}=1.5$; (b) $\alpha_{1}=2.5$; (c) $\alpha_{1}=4$.

(a)

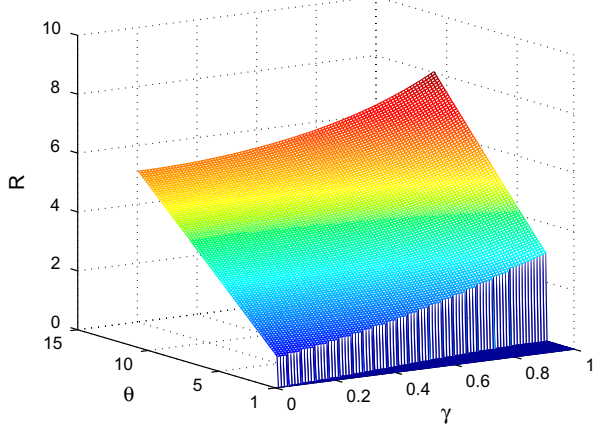

(b)

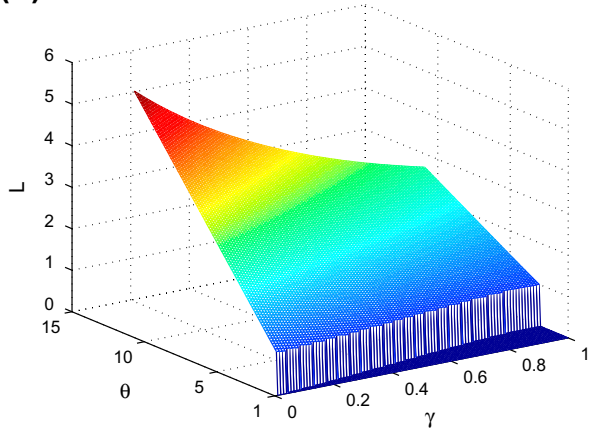

Figure 9. Plots of the functions (a) $R\left(\alpha_{0}, \gamma, \alpha_{1}\right)$; and (b) $L\left(\alpha_{0}, \gamma, \alpha_{1}\right)$, by considering $\alpha_{0}=1$.

(i) if $\alpha \leq R$, the leading firm has a higher profit than firm $F_{2}$, i.e. $\pi_{2}^{*} \leq \pi_{1}^{*}$;

(ii) if $\alpha \geq R$, firm $F_{2}$ has a higher profit than the leading firm, i.e. $\pi_{2}^{*} \geq \pi_{1}^{*}$.

(c) Case $\theta>J_{\gamma}$. The values $L, R \in\left(\alpha_{0}, \alpha_{1}\right)$. Furthermore,

(i) if $L \leq \alpha \leq R$, the leading firm has a higher profit than firm $F_{2}$, i.e. $\pi_{2}^{*} \leq \pi_{1}^{*}$;

(ii) if either $\alpha \leq L$ or $\alpha \geq R$, firm $F_{2}$ has a higher profit than the leading firm, i.e. $\pi_{2}^{*} \geq \pi_{1}^{*}$.

Theorem 4 is proved in the Appendix. This theorem allows us to say that even with small uncertainty about the demand, the follower can achieve greater profits than the leader, if their products are sufficiently differentiated (Figure 8). In Figure 9, we present the plots of $R$ and $L$ as functions of the demand uncertainty parameter $\theta$ and of the degree of differentiation $\gamma$. In Figure 10, we show the plots of $R$ and $L$ as functions of the demand uncertainty parameter $\theta$, for some chosen values of the degree $\gamma$ of differentiation. We take $\alpha_{0}=1$ and, so, we observe that $\alpha_{1}=\theta$. We note that the values $L$ and $R$ are increasing with $\theta$. 
(a)

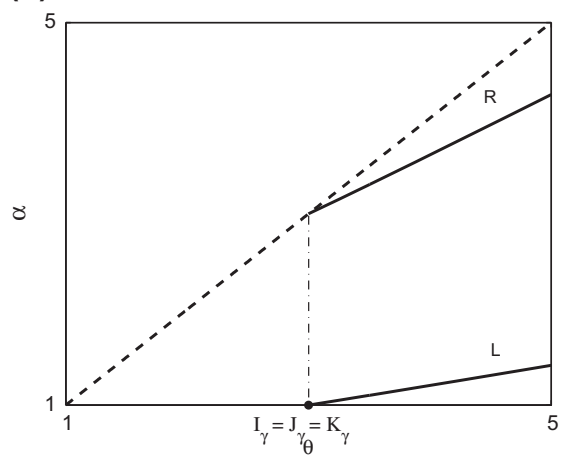

(b)

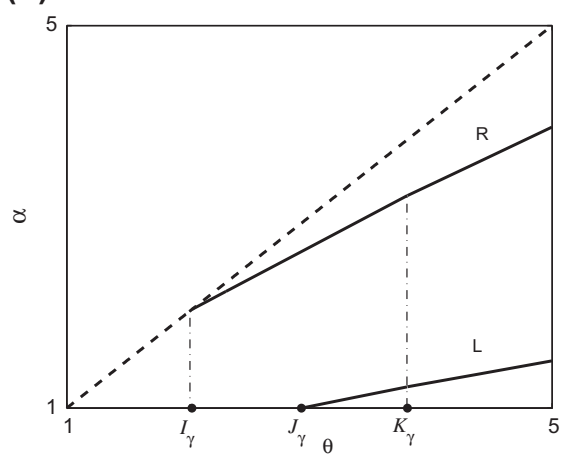

Figure 10. Plots of the functions $R\left(\alpha_{0}, \gamma, \alpha_{1}\right)$ and $L\left(\alpha_{0}, \gamma, \alpha_{1}\right)$, by considering $\alpha_{0}=1$ and in the cases of (a) $\gamma=1$; and (b) $\gamma=0.9$.

\section{Ex-ante probability of higher ex-post profits}

Now, we are going to compute, in terms of the demand uncertainty parameter $\theta$ and of the product differentiation degree $\gamma$, the ex-ante probability $P\left(\pi_{2}^{*}>\pi_{1}^{*}\right)$ of the second firm having higher ex-post profit than the leading firm. Using the results presented in Theorem 4, we get the following corollary.

Corollary 2

(a) If $\theta<I_{\gamma}$, then $P\left(\pi_{2}^{*}>\pi_{1}^{*}\right)=0$.

(b) If $I_{\gamma} \leq \theta \leq J_{\gamma}$, then

$$
P\left(\pi_{2}^{*}>\pi_{1}^{*}\right)=\frac{\left(4-3 \gamma^{2}\right) \theta-\left(4-\gamma^{2}\right)}{4\left(2-\gamma^{2}\right)(\theta-1)}
$$

(c) If $\theta \geq J_{\gamma}$, then

$$
P\left(\pi_{2}^{*}>\pi_{1}^{*}\right)= \begin{cases}\frac{\left(4-2 \gamma-\gamma^{2}\right) \theta-\left(4+2 \gamma-3 \gamma^{2}\right)}{2\left(2-\gamma^{2}\right)(\theta-1)}, & \text { if } \theta \leq K_{\gamma} \\ 1-\frac{2 \Sigma}{3 \gamma^{2}(\theta-1)}, & \text { if } \theta \geq K_{\gamma} .\end{cases}
$$

The economic interpretations of this result include: (i) given the degree of the differentiation of the goods $\gamma$, the probability $P\left(\pi_{2}^{*}>\pi_{1}^{*}\right)$ of the follower firm having higher profit than the leading firm increases with the degree of the demand uncertainty; and (ii) given the demand uncertainty, the probability $P\left(\pi_{2}^{*}>\pi_{1}^{*}\right)$ of the follower firm having higher profit than the leading firm increases with the differentiation of the goods. Furthermore, when the goods are homogeneous, the probability $P\left(\pi_{1}^{*}>\pi_{2}^{*}\right)$ of the leading firm having higher profit than the follower firm is greater than the probability of the opposite situation. However, when the goods are sufficiently different, for sufficiently high level of uncertainty, the probability $P\left(\pi_{2}^{*}>\pi_{1}^{*}\right)$ of the follower firm having higher profit than the leading firm is greater than the probability of the opposite situation (see Figure 11). 


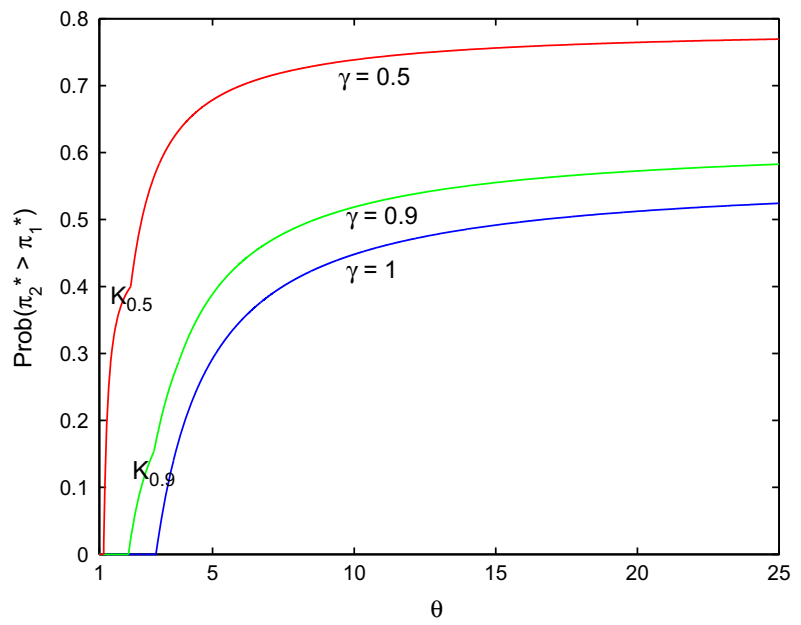

Figure 11. The probability of the second firm having higher profit than the leading firm, as a function of the demand uncertainty parameter $\theta$, for different degrees $\gamma$ of the differentiation of the goods, by taking $\alpha_{0}=1$.

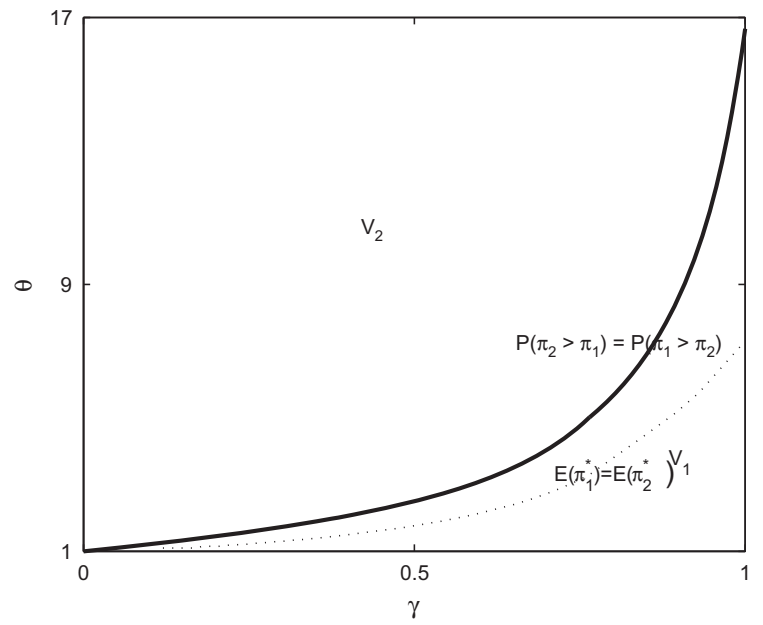

Figure 12. Solid line refers to the equality between the probabilities of the second firm having higher profit than the leading firm and the dashed line refers to the equality between the expected profits of both firms.

We observe that

$$
P\left(\pi_{2}^{*}>\pi_{1}^{*}\right)=\frac{1}{2} \Leftrightarrow \begin{cases}\theta=\frac{\gamma^{2}-\gamma-1}{\gamma-1}, & \text { if } 0 \leq \gamma \leq 3-\sqrt{5} \\ \theta=\frac{32-5 \gamma^{2}-4 \gamma \sqrt{\gamma^{2}+32}}{32-32 \gamma+3 \gamma^{2}}, & \text { if } 3-\sqrt{5} \leq \gamma \leq 1\end{cases}
$$


For a sufficiently high level of uncertainty $(\theta>9+4 \sqrt{33} / 3)$, the probability $P\left(\pi_{2}^{*}>\pi_{1}^{*}\right)$ of the follower firm having higher profit than the leading firm is greater than the probability of the opposite situation, independently of the degree $\gamma$ of differentiation. Let $V_{1}=\{(\gamma, \theta)$ : $\left.P_{1}>P_{2}\right\}$ and $V_{2}=\left\{(\gamma, \theta): P_{1}<P_{2}\right\}$. In Figure 12, we show the region where the probability of the second firm having higher profit than the leading firm is greater than the probability of the opposite situation.

We also observe that

$$
\lim _{\theta \rightarrow \infty} P\left(\pi_{2}^{*}>\pi_{1}^{*}\right)=\frac{8-\gamma^{2}-2 \sqrt{\gamma^{4}+6 \gamma^{3}-16 \gamma^{2}+16}}{3 \gamma^{2}},
$$

and

$$
\frac{7-2 \sqrt{7}}{3} \leq \frac{8-\gamma^{2}-2 \sqrt{\gamma^{4}+6 \gamma^{3}-16 \gamma^{2}+16}}{3 \gamma^{2}} \underset{\gamma \rightarrow 0}{\longrightarrow} 1 .
$$

\section{Conclusions}

In order to analyse the leadership and flexibility advantages, we considered three different situations, that depend upon the uncertainty parameter $\theta$ given by the ratio $\alpha_{1} / \alpha_{0}$ between the endpoints of the demand interval $\left[\alpha_{0}, \alpha_{1}\right]$ in which the parameter $\alpha$ is uniformly distributed. We found two functions $I_{\gamma}$ and $J_{\gamma}$, that depend upon the degree $\gamma$ of differentiation of the goods, such that (i) if $\theta<I_{\gamma}$, then the leading firm has a higher profit than the follower; (ii) if $I_{\gamma} \leq \theta \leq J_{\gamma}$, then when the realized demand is very high, the leading firm has a lower profits than the follower, otherwise the leading firm has a higher profit than the follower; and (iii) if $\theta>J_{\gamma}$, then when the realized demand is very low or very high, the leading firm has a lower profit than the follower, and when the realized demand is in an intermediate region, the leading firm has a higher profit than the follower. We observed that for homogeneous goods ( $\gamma=1$, Liu's case), the functions $I_{\gamma}$ and $J_{\gamma}$ coincide, i.e. $I_{1}=J_{1}$, and for non-homogeneous goods $(0 \leq \gamma<1)$, we have that $I_{\gamma}<J_{\gamma}$.

We showed that, when the goods are homogeneous, the probability $P\left(\pi_{1}^{*}>\pi_{2}^{*}\right)$ of the leading firm having higher profit than the follower firm is greater than the probability of the opposite situation; however, if the goods are sufficiently different or have high level of uncertainty, the probability $P\left(\pi_{2}^{*}>\pi_{1}^{*}\right)$ of the follower firm having higher profit than the leading firm is greater than one half.

\section{Acknowledgements}

This work is part-funded by the ERDF European Regional Development Fund through the COMPETE Programme (operational programme for competitiveness) and by National Funds through the FCT Fundação para a Ciência e a Tecnologia (Portuguese Foundation for Science and Technology) within project FCOMP - 01-0124-FEDER-022701. We are grateful to G. Athanasios, J.G. Brida, I. Jurado and to a couple of anonymous referees for very useful comments and suggestions on preliminary versions of this work. We are grateful for comments and suggestions from participants at 24th European Conference on Operational Research (Lisbon, Portugal, 2010), where an earlier version of this paper was presented. This work was partly funded by National Funds through the FCT Fundação para a Ciência e a Tecnologia (Portuguese Foundation for Science and Technology) within project PTDC/MAT/121107/2010. Fernanda Ferreira, Flávio Ferreira and Miguel Ferreira thank financial support from ESEIG/IPP. Alberto Pinto also thanks financial support from FCUP/UP. 
Miguel Ferreira also thanks financial support given by FCT in the form of a Pos-Doc grant with the reference SFRH/BPD/73357/2010.

\section{References}

[1] von Stackelberg H. Marktform und Gleichgewicht. Vienna: Julius Springer;1934.

[2] Liu Z. Stackelberg leadership with demand uncertainty. Manage. Decis. Econ. 2005;26:345-350.

[3] van Damme CHECK CHECK E, Hurkens S. Endogenous Stackelberg leadership. Games Econ. Behav. 1999;28:105-129.

[4] Gal-Or E. First-mover and second-mover advantages. Int. Econ. Rev. 1985;26:649-653.

[5] Gal-Or E. First-mover disadvantages with private information. Rev. Econ. Stud. 1987;54:279292.

[6] Hirokawa M. Strategic choice of quantity stickiness and Stackelberg leadership. Bull. Econ. Res. 2001;53:19-34.

[7] Hirokawa M, Sasaki D. Endogeneous co-leadership when demand is uncertain. Aust. Econ. Pap. 2000;39:278-290.

[8] Hirokawa M, Sasaki D. Endogeneously asynchronous entries into an uncertain industry. J. Econ. Manage. Strat. 2001;10:435-461.

[9] Hoppe H. The timing of new technology adoption: theoretical models and empirical evidence. Manch. Sch. 2002;70:56-76.

[10] Hoppe H, Lehmann-Grube U. Second-mover advantages in dynamic quality competition. J. Econ. Manage. Strat. 2001;10:419-433.

[11] Hoppe H, Lehmann-Grube U. Innovation timing games: a general framework with applications. J. Econ. Theory. 2005;121:30-50.

[12] Huck S, Konrad K, Muller W. Big fish eat small fish: on merger in Stackelberg markets. Econ. Lett. 2001;73:213-217.

[13] Maggi G. Endogenous leadership in a new market. RAND J. Econ. 1996;27:641-659.

[14] Pal D. Cournot duopoly with two production periods and cost differentials. J. Econ. Theory. 1991;55:441-448.

[15] Pal D, Sarkar J. A Stackelberg oligopoly with nonidentical firms. Bull. Econ. Res. 2001;53:127134.

[16] Robson A. Stackelberg and marshall. Am. Econ. Rev. 1990;80:69-82.

[17] Stalter K. Moving first on a winning idea doesn't ensure first-place finish: pioneers find pitfalls. Investor's Business Daily. 2002 Oct 7.

[18] Vives X. Duopoly information equilibrium: Cournot and Bertrand. J. Econ. Theory. 1984;34:7194.

\section{Appendix}

In this Appendix, we prove of the results presented throughout the paper.

Proof of Theorem 1 Using backwards-induction, we first compute firm $F_{2}$ 's reaction, $q_{2}^{*}\left(q_{1}, \alpha\right)$, to an arbitrary quantity $q_{1}$ fixed by firm $F_{1}$, and to the realized demand parameter $\alpha$. The quantity $q_{2}^{*}\left(q_{1}, \alpha\right)$ is given by

$$
\arg \max _{q_{2} \geq 0} q_{2}\left(\alpha-\gamma q_{1}-q_{2}\right),
$$

which yields

$$
q_{2}^{*}\left(q_{1}, \alpha\right)=\max \left\{\frac{\alpha-\gamma q_{1}}{2}, 0\right\} .
$$

Therefore, firm $F_{1}$ 's problem in the first stage of the game amounts to determine

$$
q_{1}^{*}=\arg \max _{q_{1} \geq 0} E\left(q_{1} \cdot p_{1}\right),
$$


where $p_{1}=\alpha-q_{1}-\gamma q_{2}^{*}\left(q_{1}, \alpha\right)$ and $E(\bullet)$ is the expectation with respect to the demand intercept $\alpha$. We are going to study separately the cases: (I) $\alpha_{0} \geq \gamma q_{1}$ and $(I I) \alpha_{0} \leq \gamma q_{1}$. Note that the density function of $\alpha$ 's distribution is $1 /\left(\alpha_{1}-\alpha_{0}\right)$.

Case I $\alpha_{0} \geq \gamma q_{1}$ (see Figure 13). In this case, $\alpha \geq \alpha_{0} \geq \gamma q_{1}$, and so $q_{2}^{*}\left(q_{1}, \alpha\right)=\left(\alpha-\gamma q_{1}\right) / 2$. Therefore,

$$
\begin{aligned}
E\left(q_{1}\left(\alpha-q_{1}-\gamma q_{2}^{*}\left(q_{1}, \alpha\right)\right)\right) & =\int_{\alpha_{0}}^{\alpha_{1}} q_{1}\left(\alpha-q_{1}-\gamma \frac{\alpha-\gamma q_{1}}{2}\right) \frac{1}{\alpha_{1}-\alpha_{0}} d \alpha \\
& =\frac{(2-\gamma)\left(\alpha_{1}+\alpha_{0}\right) q_{1}-2\left(2-\gamma^{2}\right) q_{1}^{2}}{4} .
\end{aligned}
$$

Then, firm $F_{1}$ 's best quantity $q_{1}^{*}$ solves the equation

$$
-4\left(2-\gamma^{2}\right) q_{1}+(2-\gamma)\left(\alpha_{1}+\alpha_{0}\right)=0 .
$$

Hence,

$$
q_{1}^{*}=\frac{(2-\gamma)\left(\alpha_{1}+\alpha_{0}\right)}{4\left(2-\gamma^{2}\right)}
$$

and so

$$
q_{2}^{*}=\frac{\alpha}{2}-\frac{\gamma(2-\gamma)\left(\alpha_{1}+\alpha_{0}\right)}{8\left(2-\gamma^{2}\right)} .
$$

We observe that the value $q_{1}^{*}$ obtained in (4) satisfies the hypothesis $\alpha_{0} \geq \gamma q_{1}$ considered in Case I if, and only if, $\theta \leq K_{\gamma}$.

Case II $\alpha_{0} \leq \gamma q_{1}$ (see Figure 14). In this case,

(i) if $\alpha \leq \gamma q_{1}$, then $q_{2}^{*}\left(q_{1}, \alpha\right)=0$; and

(ii) if $\alpha \geq \gamma q_{1}$, then $q_{2}^{*}\left(q_{1}, \alpha\right)=\left(\alpha-\gamma q_{1}\right) / 2$.

Therefore,

$$
\begin{aligned}
E\left(q_{1}\left(\alpha-q_{1}-\gamma q_{2}^{*}\left(q_{1}, \alpha\right)\right)\right)= & \int_{\alpha_{0}}^{\gamma q_{1}} q_{1}\left(\alpha-q_{1}\right) \frac{1}{\alpha_{1}-\alpha_{0}} d \alpha \\
& +\int_{\gamma q_{1}}^{\alpha_{1}} q_{1}\left(\alpha-q_{1}-\gamma \frac{\alpha-\gamma q_{1}}{2}\right) \frac{1}{\alpha_{1}-\alpha_{0}} d \alpha \\
= & \frac{\left((2-\gamma) \alpha_{1}^{2}-2 \alpha_{0}^{2}\right) q_{1}-2\left(\left(2-\gamma^{2}\right) \alpha_{1}-2 \alpha_{0}\right) q_{1}^{2}-\gamma^{3} q_{1}^{3}}{4\left(\alpha_{1}-\alpha_{0}\right)} .
\end{aligned}
$$

Then, firm $F_{1}$ 's best quantity $q_{1}^{*}$ solves the equation

$$
-3 \gamma^{3} q_{1}^{2}-4\left(\left(2-\gamma^{2}\right) \alpha_{1}-2 \alpha_{0}\right) q_{1}+(2-\gamma) \alpha_{1}^{2}-2 \alpha_{0}^{2}=0 .
$$

Hence,

$$
q_{1}^{*}=\frac{\Delta}{3 \gamma^{3}},
$$

and so

$$
q_{2}^{*}=\left\{\begin{array}{ll}
0, & \text { if } \alpha<\Delta /\left(3 \gamma^{2}\right) \\
\frac{\alpha}{2}-\frac{\Delta}{6 \gamma^{2}}, & \text { if } \alpha \geq \Delta /\left(3 \gamma^{2}\right)
\end{array} .\right.
$$

We observe that the value $q_{1}^{*}$ obtained in (7) satisfies the hypothesis $\alpha_{0} \leq \gamma q_{1}$ considered in Case II if, and only if, $\theta \geq K_{\gamma}$. 


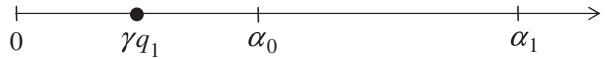

Figure 13. $\alpha_{0} \geq \gamma q_{1}($ Case I).

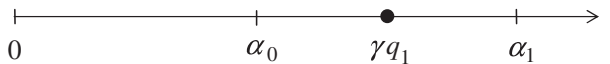

Figure 14. $\alpha_{0} \leq \gamma q_{1}($ Case II).

Case I

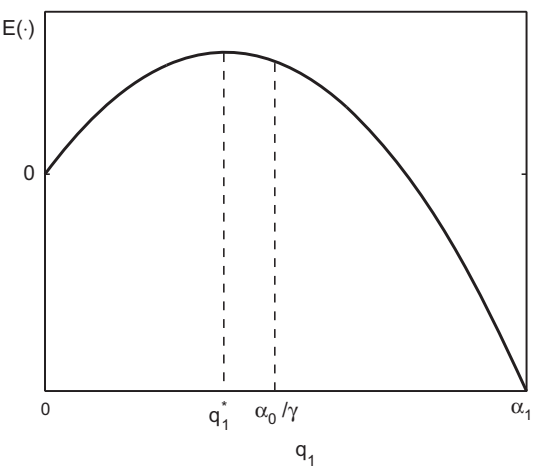

Case II

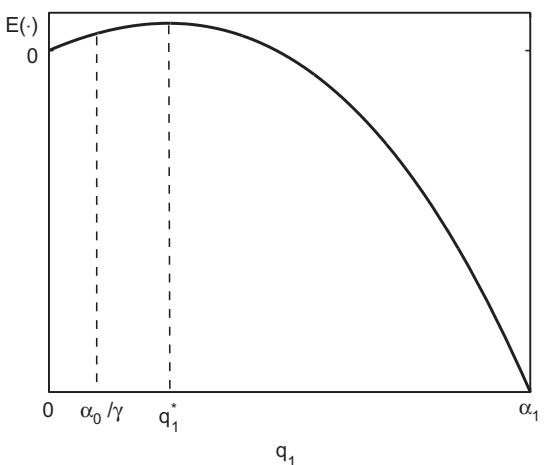

Figure 15. Case I $\left(\theta \leq K_{\gamma}\right)$ : $\arg \max _{q_{1} \geq 0} E\left(q_{1} \cdot p_{1}\right) \leq \alpha_{0} / \gamma$; Case II $\left(\theta \geq K_{\gamma}\right)$ : $\arg \max _{q_{1} \geq 0} E\left(q_{1} \cdot p_{1}\right) \geq \alpha_{0} / \gamma$.

We note that, in Case I $\left(\theta \leq K_{\gamma}\right)$ we have that $\arg \max _{q_{1} \geq 0} E\left(q_{1} \cdot p_{1}\right) \leq \alpha_{0} / \gamma$, and in Case II $\left(\theta \geq K_{\gamma}\right)$ we have that $\arg \max _{q_{1} \geq 0} E\left(q_{1} \cdot p_{1}\right) \geq \alpha_{0} / \gamma$ (see Figure 15).

Proof of Corollary 1 First, suppose that $\theta \leq K_{\gamma}$. By Theorem 1, we get that

$$
q_{1}^{*}-q_{2}^{*}=\frac{4-\gamma^{2}}{8\left(2-\gamma^{2}\right)}\left(\alpha_{1}+\alpha_{0}\right)-\frac{\alpha}{2} .
$$

Therefore, $q_{1}^{*}-q_{2}^{*} \geq 0$ if, and only if,

$$
\alpha \leq \frac{4-\gamma^{2}}{4\left(2-\gamma^{2}\right)}\left(\alpha_{1}+\alpha_{0}\right) .
$$

Since $\frac{4-\gamma^{2}}{4\left(2-\gamma^{2}\right)}\left(\alpha_{1}+\alpha_{0}\right)>\alpha_{1}$ if, and only if, $\theta<I_{\gamma}$, and $I_{\gamma} \leq k_{\gamma}$, we get the statement (a). Since $\frac{4-\gamma^{2}}{4\left(2-\gamma^{2}\right)}\left(\alpha_{1}+\alpha_{0}\right) \in\left[\alpha_{0}, \alpha_{1}\right]$ if, and only if, $\theta \geq I_{\gamma}$, we get the statement (b).

Now, suppose that $\theta \geq K_{\gamma}$. By Theorem 1, we get that

- if $\alpha<\Delta /\left(3 \gamma^{2}\right)$, then

$$
q_{1}^{*}-q_{2}^{*}=q_{1}^{*}=\frac{\Delta}{3 \gamma^{3}},
$$

which is positive; 
- if $\alpha \geq \Delta /\left(3 \gamma^{2}\right)$, then

$$
q_{1}^{*}-q_{2}^{*}=\frac{(2+\gamma) \Delta}{6 \gamma^{3}}-\frac{\alpha}{2} .
$$

Therefore, $q_{1}^{*}-q_{2}^{*} \geq 0$ if, and only if,

$$
\alpha \leq \frac{(2+\gamma) \Delta}{3 \gamma^{3}}
$$

which implies the statement (c).

Proof of Theorem 2 Firm $F_{1}$ 's expected profit, $E\left(\pi_{1}^{*}(\alpha)\right.$ ), is obtained by (3) and (6). Firm $F_{2}$ 's expected profit, $E\left(\pi_{2}^{*}(\alpha)\right)$, is determined by

$$
E\left(\pi_{2}^{*}(\alpha)\right)=\int_{\alpha_{0}}^{\alpha_{1}} q_{2}^{*}\left(\alpha-\gamma q_{1}^{*}-q_{2}^{*}\right) \frac{1}{\alpha_{1}-\alpha_{0}} d \alpha,
$$

with $q_{1}^{*}$ and $q_{2}^{*}$ given, respectively, by (4) and (5), in the case of $\theta \leq K_{\gamma}$, and given, respectively, by (7) and (8), in the case of $\theta \geq K_{\gamma}$. Finally, we note that the values $(\theta, \gamma)$ in the set $P$ are the ones that satisfy $\theta \leq K_{\gamma}$, and the values $(\theta, \gamma)$ in the set $Q$ are the ones that satisfy $\theta \geq K_{\gamma}$.

Proof of Theorem 3 Here, we compute the exact value $\theta_{0}$, when we take $\alpha_{0}=1$ and $\gamma=1$, such that if the uncertainty parameter $\theta$ is greater than $\theta_{0}$, then the expected profit of the follower firm is always greater than the expected profit of the leading firm.

Let

$$
\begin{gathered}
D=\frac{10348768573}{5971968}-\frac{8804787277 \sqrt{17}}{23887872}, E=\frac{10348768573}{5971968}+\frac{8804787277 \sqrt{17}}{23887872}, \\
F=\frac{118172131}{995328}-\frac{10655929 \sqrt{17}}{373248} \text { and } G=\frac{118172131}{995328}+\frac{10655929 \sqrt{17}}{373248} .
\end{gathered}
$$

The value $\theta_{0}$ is given by

$$
\begin{aligned}
& \theta_{0}= \frac{9}{4}+\sqrt{\frac{143}{48}-\left(\frac{3539}{3456}-\frac{3011 \sqrt{17}}{13824}\right)^{1 / 3}-\left(\frac{3539}{3456}+\frac{3011 \sqrt{17}}{13824}\right)^{1 / 3}} \\
&+\sqrt{\frac{143}{24}+\left(\frac{3539}{3456}-\frac{3011 \sqrt{17}}{13824}\right)^{1 / 3}+\left(\frac{3539}{3456}+\frac{3011 \sqrt{17}}{13824}\right)^{1 / 3}+\sqrt{H}},
\end{aligned}
$$

where

$$
H=D^{1 / 3}+E^{1 / 3}+F^{1 / 3}+G^{1 / 3}+\frac{19013}{576} .
$$

Therefore, we observe that $\theta_{0} \approx 7.30$.

Proof of Theorem 4 By Theorem 1, in the case where $\theta \leq K_{\gamma}$, the profits $\pi_{1}^{*}$ and $\pi_{2}^{*}$ at equilibrium are given by

$$
\pi_{1}^{*}=\frac{(2-\gamma)^{2}\left(4 \alpha-\alpha_{1}-\alpha_{0}\right)\left(\alpha_{1}+\alpha_{0}\right)}{32\left(2-\gamma^{2}\right)}
$$

and

$$
\pi_{2}^{*}=\frac{\left(4 \alpha\left(2-\gamma^{2}\right)-\gamma(2-\gamma)\left(\alpha_{1}+\alpha_{0}\right)\right)^{2}}{64\left(2-\gamma^{2}\right)^{2}}
$$


So, we have that $\pi_{1}^{*}-\pi_{2}^{*}>0$ if, and only if,

$$
L_{1} \equiv \frac{(2-\gamma)^{2}}{4\left(2-\gamma^{2}\right)}\left(\alpha_{0}+\alpha_{1}\right)<\alpha<\frac{4-\gamma^{2}}{4\left(2-\gamma^{2}\right)}\left(\alpha_{0}+\alpha_{1}\right) \equiv R_{1} .
$$

Again by Theorem 1 , in the case of $\theta \geq K_{\gamma}$, the profits $\pi_{1}^{*}$ and $\pi_{2}^{*}$ at equilibrium are given by

$$
\pi_{1}^{*}= \begin{cases}\frac{\left(3 \alpha \gamma^{3}-\Delta\right) \Delta}{9 \gamma^{6}}, & \text { if } \alpha<\Delta /\left(3 \gamma^{2}\right) \\ \frac{\left(3 \alpha \gamma^{3}(2-\gamma)-\left(2-\gamma^{2}\right) \Delta\right) \Delta}{18 \gamma^{6}}, & \text { if } \alpha \geq \Delta /\left(3 \gamma^{2}\right)\end{cases}
$$

and

$$
\pi_{2}^{*}= \begin{cases}0, & \text { if } \alpha<\Delta /\left(3 \gamma^{2}\right) \\ \frac{\left(3 \alpha \gamma^{2}-\Delta\right)^{2}}{36 \gamma^{4}}, & \text { if } \alpha \geq \Delta /\left(3 \gamma^{2}\right)\end{cases}
$$

So, we have that $\pi_{1}^{*}-\pi_{2}^{*}>0$ if, and only if,

$$
L_{2} \equiv \frac{(2-\gamma) \Delta}{3 \gamma^{3}}<\alpha<\frac{(2+\gamma) \Delta}{3 \gamma^{3}} \equiv R_{2}
$$

(a) For $\theta<I_{\gamma}$, we have that $L_{1}<\alpha_{0}$ and $R_{1}>\alpha_{1}$. Therefore, $\pi_{2}^{*}<\pi_{1}^{*}$ for all $\alpha \in\left[\alpha_{0}, \alpha_{1}\right]$.

(b) For $I_{\gamma} \leq \theta \leq J_{\gamma}$, we have that $L_{1}<\alpha_{0}$ and $\alpha_{0} \leq R_{1} \leq \alpha_{1}$. Therefore, if $\alpha<R_{1}$ then $\pi_{2}^{*}<\pi_{1}^{*}$; and if $\alpha>R_{1}$ then $\pi_{2}^{*}>\pi_{1}^{*}$.

(c) For $J_{\gamma}<\theta \leq K_{\gamma}$, we have that $\alpha_{0} \leq L_{1}, R_{1} \leq \alpha_{1}$. Therefore, if $L_{1}<\alpha<R_{1}$ then $\pi_{2}^{*}<\pi_{1}^{*}$; and if either $\alpha<L_{1}$ or $\alpha>R_{1}$ then $\pi_{2}^{*}>\pi_{1}^{*}$.

For $\theta>K_{\gamma}$, we have that $\alpha_{0} \leq L_{2}, R_{2} \leq \alpha_{1}$. Therefore, if $L_{2}<\alpha<R_{2}$ then $\pi_{2}^{*}<\pi_{1}^{*}$; and if either $\alpha<L_{2}$ or $\alpha>R_{2}$ then $\pi_{2}^{*}>\pi_{1}^{*}$. 